\title{
Development of the Catecholamine Innervation of the Supraoptic Nucleus in the Brattleboro Rat ${ }^{1}$
}

\author{
JOHN R. SLADEK, JR., ${ }^{2}$ JULIE FIELDS, CAROL J. PHELPS \\ AND HENRY KHACHATURIAN \\ Department of Anatomy and Center for Brain Research, University of Rochester School of Medicine \\ Rochester, NY 14642 \\ and Mental Health Research Institute, University of Michigan School of Medicine, Ann Arbor, MI 48109
}

\begin{abstract}
SLADEK, J. R., JR., J. FIELDS, C. J. PHELPS AND H. KHACHATURIAN. Development of the catecholamine innervation of the supraoptic nucleus in the Brattleboro rat. PEPTIDES 5: Suppl 1, 151-155, 1984.-The ontogenetic development of the noradrenergic innervation of the supraoptic nucleus was studied in the Brattleboro rat at late postcoital and early postnatal ages. This genetic mutant offers a useful model for analysis of neuronal development because of the absence of a specific peptide component of identifiable target neurons and has been used presently to eliminate the possibility that such substances are essential for the establishment of normal connectivity during postnatal development. In this model, catecholamine varicosities were seen in juxtaposition to vasopressin-deficient perikarya during the initial phases of postnatal development, but these varicosities gradually decreased in number suggesting the possibility that the target neuron peptide, or some functional aspect of the neuron, may be necessary for the normal maintenance of this neuronal interaction.
\end{abstract}

$\begin{array}{llll}\begin{array}{l}\text { Noradrenaline } \\ \text { Histofluorescence }\end{array} & \begin{array}{c}\text { Supraoptic nucleus Ontogeny } \\ \text { Immunohistochemistry }\end{array} & \text { Brattleboro rat Neurophysin } & \text { Vasopressin-deficient }\end{array}$

THE developing hypothalamus is characterized by a relatively early onset of peptide biosynthesis in neurons of the supraoptic (SON) and paraventricular nuclei $[9,11]$. The appearance of catecholamines within varicosities in these same nuclei follows shortly thereafter [5]. It is clear that one catecholamine, noradrenaline, plays a role in the regulation of vasopressin release from neurons of the SON as determined from several lines of physiological evidence $[1,6,11]$. Although the precise role played by noradrenaline may be dependent on experimental and in situ parameters it equally is clear that the morphology of this neurochemically-rich region of the hypothalamus favors a noradrenaline-vasopressin interaction. Simultaneous comparator bridge analysis of catecholamine histofluorescence and neurophysin immunohistochemistry has confirmed the existence of an innervation pattern which heavily favors vasopressin perikarya in both rat and monkey hypothalamus [7,14]. That this innervation pattern is due largely to noradrenaline has been confirmed with immunohistochemical analysis of catecholamine synthetic enzymes [19].

Many cues have been hypothesized as responsible for directing or controlling the establishment of neuronal inter- actions during pre and postnatal development. These include various trophic or guidance factors which may exist on postsynaptic membranes. For example, Hemmendinger and colleagues have demonstrated that dopamine neurons fail to form axonal outgrowths, but do form dendritic arbors, in the absence of appropriate target neurons when grown in tissue culture [43]. Whether a peptide, such as vasopressin or its propressophysin precursor, is essential for the normal establishment of interactions has not been tested. Our previous study on the co-development of catecholamine varicosities and neurophysin-positive neurons in the rat hypothalamus revealed moderate to strong neurophysin staining prior to the demonstration of catecholamine varicosity fluorescence [5]. This suggested the possibility, based on temporal occurence, that the target neuron product might be involved in the ingrowth of noradrenergic axons and subsequently the establishment of appropriate neuronal connectivity.

In order to test the above question, the development of noradrenergic fibers in the SON was studied in the Brattleboro (DI) rat, a genetic mutant which exhibits a profound diabetes insipidus due to a failure of extant magnocellular neurons to synthesize vasopressin or its associated

\footnotetext{
'A portion of this work was presented at the Fifth Brain-Endocrine Interaction Symposium in Wurzburg, West Germany, July 1983, on the authors' behalf by Dr. Carol Phelps of the Department of Anatomy, University of Rochester School of Medicine.

${ }^{2}$ Requests for reprints should be addressed to John R. Sladek, Jr., Professor and Chairman of Anatomy, Department of Anatomy, Box 603, University of Rochester School of Medicine, Rochester, NY 14642.
} 


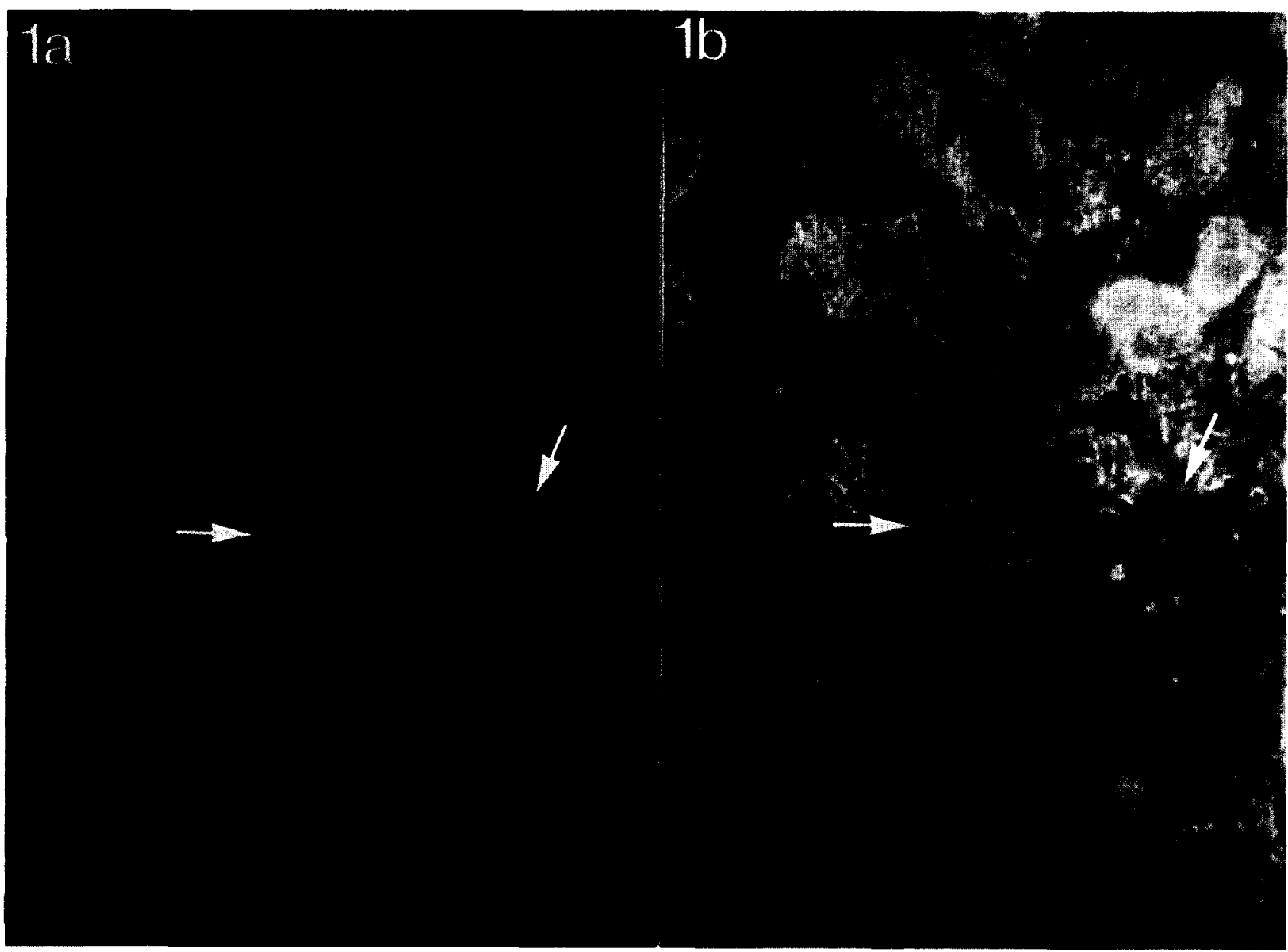

FIG. 1.

FIGS. 1-3. Each figure depicts the distribution of developing catecholamine fibers in relation to oxytocin-positive and vasopressin-deficient magnocellular neurons in a similar portion of the supraoptic nucleus ( $\mathrm{SON}$ ) in the Brattleboro rat at three postnatal ages. Panel $\mathrm{A}$ is catecholamine histofluorescence. panel $B$ is a comparator bridge view which adds neurophysin staining from the adjacent section to the fluorescence depicted in $A$. The arrows indicate catecholamine varicosities which appear juxtaposed to vasopressin-deficient neurons in the ventral portion of the SON. These neurons appear non-fluorescent in panel A. their nuclei are seen as dark ovals. Ages: Fig. 1, 7 days postnatal (dpn): Fig. 2, 14 dpn: Fig. 3, 21 dpn. $\times 450$.

neurophysin [20]. However, because the neurons are present in the hypothalamus they remain as targets for noradrenergic fibers. Thus the DI rat is a useful model for studying the development of neuronal interactions in the absence of a specific target neuron substance.

\section{METHOD}

Timed-pregnant rats were purchased from Blue Spruce Farms for prenatal and early postnatal analysis: for all postnatal studies past 1 day of age pups were transported with their mothers to the laboratory. Three animals were examined at each of the following ages: 19 days postcoitus and $1,7,14$ and 21 days postnatal (dpn). Brains were collected for formaldehyde-induced fluorescence of catecholamines and were processed sequentially through freezedrying, p-formaldehyde vapor perfusion and paraffin embedding as detailed previously [5]. Alternate sections were stained (a) with cresyl violet and luxol fast blue for anatomical orientation, and (b) immunohistochemically for rat neurophysin (provided by Dr. Alan G. Rohinson). Sec- tions adjacent to those stained for neurophysin were examined for catecholamine histofluorescence.

Sections were analyzed in a Leitz Orthoplan comparator bridge microscope as described earlier $[8,16]$. This permitted the following. crucial observations. Sections stained for neurophysin revealed the position of oxytocin neurons in the SON: non-stained magnocellular neurons therefore were considered as vasopressin-deficient. Adjacent sections were examined following accurate alignment in the comparator bridge system. Parts of the same neuron were seen on each section and comparative. qualitative analysis was made of (a) the relative occurrence of catecholamine varicosties within the boundaries of the SON at each age, and (b) the relative degree to which catecholamine varicosities appeared in juxtaposition to oxytocin-positive and vasopressin-free magnocellular perikarya.

RESULTS

General Observations

Catecholamine fluorescence appeared in the developing 


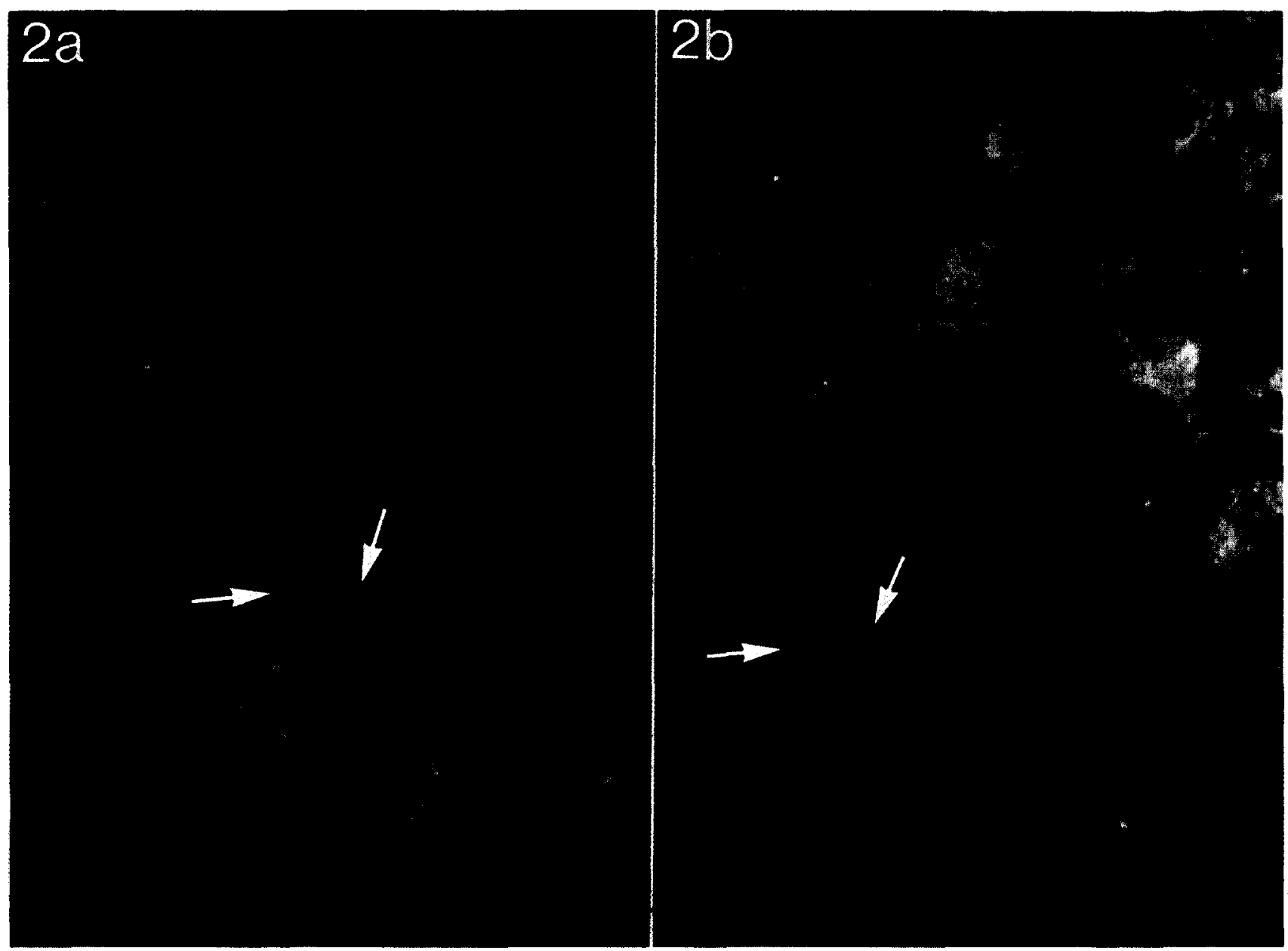

FIG. 2.

brain in the form of fine-sized, intensely fluorescent varicosities. Varicosities were punctate and this distinct fluorescence permitted visualization of varicosity position relative to magnocellular perikarya and, at later postnatal ages, proximal dendrite stumps. A general increase in varicosity density within the SON accompanied the advance in postnatal age, but the pattern stabilized abruptly and eventually declined by 21 dpn.

Neurophysin immunostaining resulted in the clear depiction of magnocellular perikarya, primarily in the rostral and dorsal portions of the SON. These corresponded positionally, to oxytocin neurons as reported previously by several laboratories. Magnocellular neurons in the caudal and ventral portions of the SON were not stained for neurophysin and therefore corresponded to vasopressin neurons as seen in normal rats. The distinction between these two groups was clear and allowed the determination of the occurrence of catecholamine varicosities in apposition to developing magnocellular perikarya as described below.

\section{Davs Postcoitus}

At this time point the SON was not well organized. Some magnocellular neurons were stained very lightly for neurophysin. Catecholamine varicosities were seen rarely and no consistent evidence was found to suggest an alignment with magnocellular perikarya.
$1 \mathrm{dph}$

Neurophysin staining appeared considerably more dense than that seen at 19 days postcoitus. Numerous neurons were seen in the dorsal and rostral portions of the SON and clearly were discernable from the peptide-deficient neurons in the ventral and caudal portions of the nucleus. Catecholamine varicosties were more prominent than seen earlier and many appeared in apposition to perikarya in the ventral SON. Varicosities were extremely scarce in the dorsal, oxytocin-rich area. Overall varicosity density was low which made it necessary to optically dissect the tissue section by sequential focusing through different planes to visualize an appreciable number of these fine-sized varicosities in juxtaposition to magnocellular perikarya. Neurons in the dorsal SON were contacted less frequently by fluorescent varicosities than those ventrally.

\section{$7 d p n$}

A sharp increase was seen in the number of varicosities in the ventral extreme of the SON, particularly ventral to the nucleus proper in the so-called basal glial lamina. This increased density extended into the ventral zone of the SON which was filled with vasopressin-free perikarya as judged by an absence of neurophysin staining. Here, numerous varicosities were seen in apposition to magnocellular perikarya (Fig. 1). The pattern dorsally was less dense. but was charac- 


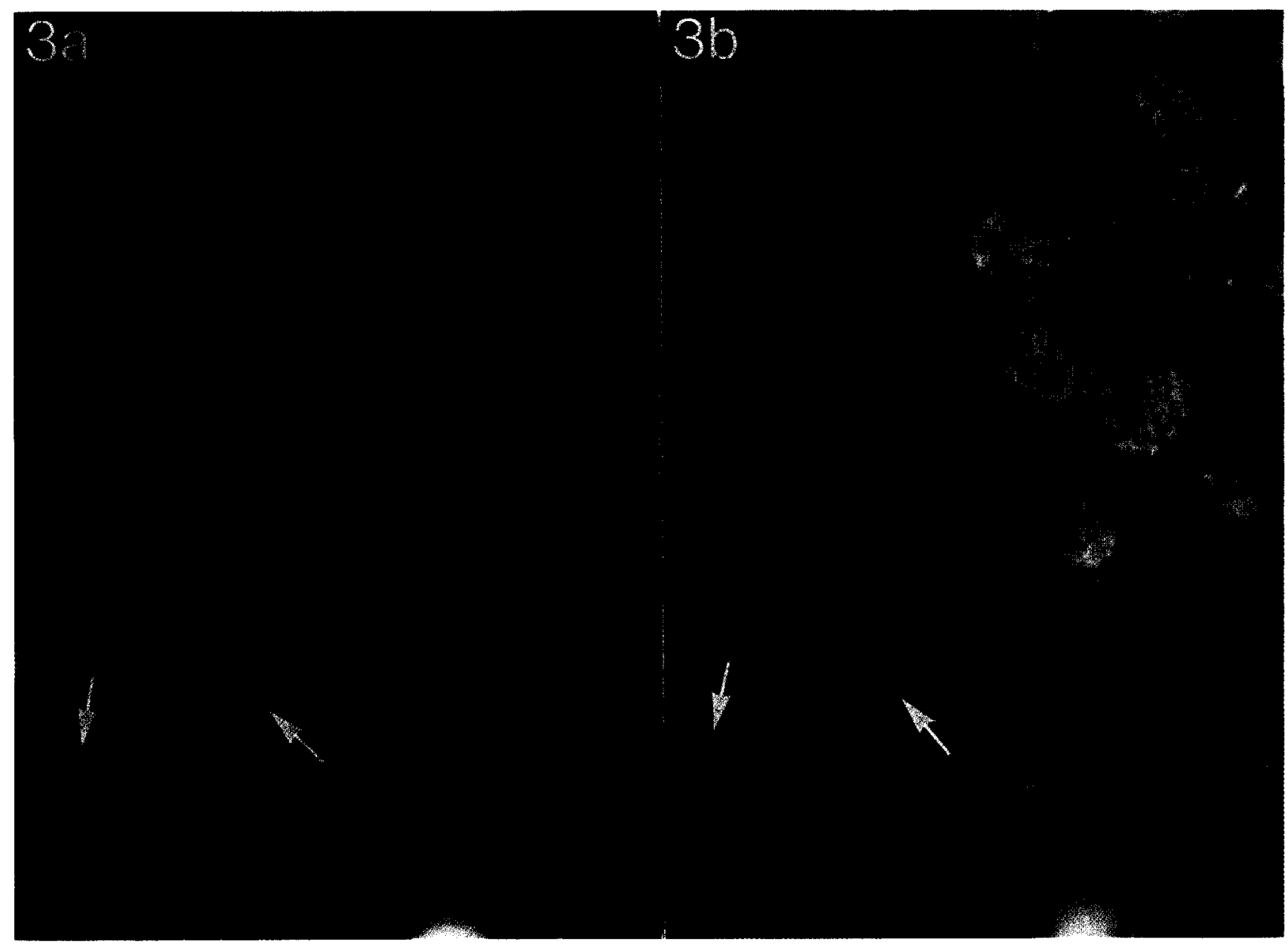

FIG. 3.

terized by varicosity-perikaryal appositions on a few neurons per section. The mid dorsal-ventral portion of the SON was characterized by an appreciable decline in varicosity density which persisted throughout later developmental ages.

Neurophysin staining was dense in a majority of perikarya in the oxytocin-rich portion of the nucleus and facilitated comparator bridge analysis.

\section{$14 d p n$}

Catecholamine varicosities in the basal lamina appeared reduced in number in comparison to the pervious age. Magnocellular neurons in the ventral SON still appeared to be contacted by varicosities (Fig. 2). Neurons in the dorsal SON frequently were seen in juxtaposition to catecholamine varicosities, although the majority of neurophysin-positive neurons did not reveal this juxtapositional phenomenon. The overall density of varicosities in the dorsal SON appeared increased in comparison to the previous age.

Neurophysin staining was very dense in the majority of dorsally-placed neurons.

\section{$21 d p n$}

The density of catecholamine varicosities in the developing SON had stabilized at this postnatal age in contradistinction to the continued increase in density reported earlier [4].
In some animals this pattern appeared to show a reduction in density over the vasopressin-deficient regions which was most apparent when viewing the SON with low power, scanning magnifications.

\section{DISCUSSION}

The present results clearly show during postnatal development that catecholamine-containing varicosities appear in juxtaposition to appropriate target neurons in the absence of biosynthesis of a particular peptide by the target neuron. This results in a relatively normal pattern of catecholamine varicosities in the SON of the DI rat through 14 days of postnatal development. The density of this pattern is reduced by $21 \mathrm{dpn}$ and is severely diminished in the adult [9]. Thus, other factors, or chemical messengers, might be involved in the development of appropriate, noradrenergic connections. One possible candidate is dynorphin, which has been shown by Watson and colleagues to coexist in vasopressin neurons in normal rat and to be present in vasopressin-deficient neurons in the DI rat [21]. Other possibilities include extracellular matrix macromolecules such as glycoproteins [4] electrical activity of the target perikarya as well as other, yet undefined, guidance cues or trophic factors.

Although the present data do not support the concept that vasopressin plays a role in the guidance of noradrenergic axons to targets in the SON, vasopressin may be important 
in the maintenance of this afferent system. This concept is based on a series of qualitative assessments of the innervation pattern as follows. The noradrenaline innervation pattern is reduced dramatically in the adult DI rat [9] and is diminished appreciably in the aged (20 to 30 month old) Fisher 344 rat $[13,17,18]$. In the latter instance, vasopressin, but neither oxytocin nor neurophysin staining was reduced in perikarya of the SON and paraventricular nucleus. The potential that these concomitant changes are related causally is supported by two additional findings. Our preliminary findings in a genetic mutant mouse which has an overabundance of vasopressin, revealed an increase in the density of the noradrenaline innervation pattern in comparison to controls [2]. Similarly, the SON of the spontaneously hyperten- sive rat is characterized by a dramatic increase in noradrenaline varicosities and vasopressin-associated neurophysin staining in magnocellular perikarya [14]. Taken together these data suggest a direct relationship between vasopressin and noradrenaline content as detected histologically which varies with changes in the physiological status of the system.

\section{ACKNOWLEDGEMENT}

This work was supported by the National Science Foundation through grant BNS 8206061 to JRS. We are grateful to Dr. Alan Robinson for a generous supply of rat neurophysin antiserum provided under USPHS grant AM 16166.

\section{REFERENCES}

1. Barker, J. L., J. W. Crayton and R. A. Nicoll. Noradrenaline and acetylcholine responses of supraoptic neurosecretory cells. $J$ Physiol (Lond) 218: 19-31, 1971.

2. Davis, B. J., H. W. Sokol and J. R. Sladek, Jr. Altered noradrenergic innervation of the supraoptic nucleus in the nephrogenic, diabetes insipidus mouse. Anat Rec 205: 44A, 1983.

3. Gottlieb, D. I. and L. Glaser. Cellular recognition during neural development. Annu Rev Neurosci 3: 303-318, 1980.

4. Hemmendinger, L. M., B. B. Garber, P. C. Hoffman and A. Heller. Target neuron-specific process formation by embryonic mesencephalic dopamine neurons in vitro. Proc Natl Acad Sci USA 78: 1264-1268, 1981.

5. Khachaturian. H. and J. R. Sladek, Jr. Simultaneous monoamine histofluorescence and neuropeptide immunocytochemistry: II. Ontogeny of catecholamines and neurophysin in the rat supraoptic and paraventricular nuclei. Peptides 1: 77-95, 1980.

6. Kimura, T., L. Share, B. C. Wang and J. T. Crofton. The role of central adrenoreceptors in the control of vasopressin release and blood pressure. Endocrinology 108: 1829-1836, 1981.

7. McNeill, T. H. and J. R. Sladek, Jr. Simultaneous monoamine histofluorescence and neuropeptide immunocytochemistry: II. Correlative distribution of catecholamine varicosities and magnocellular neurosecretory neurons in the rat supraoptic and paraventricular nuclei. J Comp Neurol 193: 1023-1033, 1980

8. McNeill, T. H. and J. R. Sladek, Jr. Simultaneous monoamine histofluorescence and neuropeptide immunocytochemistry. V. A methodology for examining correlative monoamineneuropeptide neuroanatomy. Brain Res Bull 5: 599-608, 1980.

9. Schöler, J. and J. R. Sladek, Jr. Supraoptic nucleus of the Brattleboro rat has an altered noradrenergic input. Science 214: $347-349,1981$

10. Sinding. C., A. G. Robinson, S. M. Seif and P. G. Schmid. Neurohypophyseal peptides in the developing rat fetus. Brain Res 195: 177-186, 1980.

11. Sladek. C. D. Regulation of vasopressin release by neurotransmitters, neuropeptides and osmotic stimuli. Prog Brain Res 60: 71-90, 1983.

12. Sladek, C. D., D. M. Gash, H. Khachaturian, D. E. Scott and J. R. Sladek, Jr. Maturation of the supraoptic nucleus: A multidisciplinary analysis. Peptides 1: Suppl 1, 51-67, 1980.
13. Sladek, J. R., Jr. Aging of central neuropeptide systems. Psychopharmacol Bull 19: 317-319, 1983.

14. Sladek, J. R., Jr. and C. D. Sladek. Histochemical analysis of vasopressin-neurophysin and norepinephrine in the supraoptic and paraventricular nuclei of the spontaneously hypertensive rat. Soc Neurosci Abstr 9: 546, 1983.

15. Sladek, J. R., Jr. and E. A. Zimmerman. Simultaneous monoamine histofluorescence and neuropeptide immunocytochemistry VI: Catecholamine innervation of vasopressin and oxytocin neurons in the rhesus monkey hypothalamus. Brain Res Bull 9: 431-440, 1983.

16. Sladek, J. R., Jr., C. D. Sladek, T. H. McNeill and J. G. Wood New sites of monoamine localization in the endocrine hypothal amus as revealed by new methodological approaches. In: Neural Hormones and Reproduction, Brain-Endocrine Interaction III, edited by D. E. Scott, G. P. Kozlowski and A. Weindl. Basel: Karger, 1978, pp. 154-171.

17. Sladek, J. R., Jr., H. Khachaturian, G. E. Hoffman and J. Scholer. Aging of central endocrine neurons and their aminergic afferents. Peptides 1: Suppl 1, 141-157, 1980.

18. Sladek, J. R., Jr., J. Schöler and W. E. Armstrong. Norepinephrine-vasopressin interactions during aging. In Structure and Function of Peptidergic and Aminergic Neurons, edited by Y. Sano, Y. Ibata and E. A. Zimmerman. Tokyo: Japan Scientific Soc. Press, 1983, pp. 289-298.

19. Swanson, L. W., P. E. Sawchenko, A. Berod, B. K. Hartman, K. B. Helle and D. E. Van Orden. An immunohistochemical study of the organization of catecholaminergic cells and terminal fields in the paraventricular and supraoptic nuclei of the hypothalamus. J Comp Neurol 196: 271-285, 1981.

20. Vatlin, H. The discovery of the Brattleboro rat, recommended nomenclature and the question of proper controls. Ann NY Acad Sci 394: 1-9, 1982.

21. Watson, S. J., H. Akil, W. Fischli, A. Goldstein, E. Zimmerman, G. Nilaver and T. B. van Wimersma Greidanus. Dynorphin and vasopressin: common localization in magnocellular neurons. Science 216: 85-87, 1982. 